\title{
Stimulation in Hippocampal Region CA1 in Behaving Rats Yields Long-Term Potentiation when Delivered to the Peak of Theta and Long-Term Depression when Delivered to the Trough
}

\author{
James M. Hyman, ${ }^{1}$ Bradley P. Wyble, ${ }^{1,2}$ Vikas Goyal, ${ }^{1,2}$ Christina A. Rossi, ${ }^{1}$ and Michael E. Hasselmo ${ }^{1}$ \\ ${ }^{1}$ Center for Memory and Brain, Department of Psychology, Boston University, Boston, Massachusetts 02215, and ${ }^{2}$ Department of Psychology, Harvard \\ University, Cambridge, Massachusetts 02138
}

\begin{abstract}
Experimental evidence suggests that the hippocampal theta rhythm plays a critical role in learning. Previous studies have shown longterm potentiation (LTP) to be preferentially induced with stimulation on the peak of local theta rhythm in region CA1 in anesthetized rats and with stimulation of the perforant path at the peak of theta in both anesthetized and behaving animals. We set out to determine the effects of tetanic burst stimulation in stratum radiatum of region CA1 in awake behaving animals, delivered during either the peak or the trough of the theta rhythm in the EEG. Bursts delivered to the peak resulted in an increase of $17.9 \pm 0.94 \%$ in potential slope. When identical stimulation bursts were delivered to the trough of local theta waves, the potential slope decreased $12.9 \pm 1.03 \%$. This is the first report of LTP being preferentially induced at the peak of local theta rhythm in behaving animals in region CA1 and that LTD was found in response to tetanic stimulation at the trough of the local theta wave. The results are discussed within the framework of a recent theory that proposes that the theta rhythm sets the dynamics for alternating phases of encoding and retrieval (Hasselmo et al., 2002).
\end{abstract}

Key words: theta rhythm; LTP; hippocampus; LTD; CA1; plasticity

\section{Introduction}

Both long-term potentiation (LTP) and theta rhythm in the hippocampus have been studied extensively for their role in memory formation independently, but only a few studies have examined how these two phenomena interact. Experimentally, LTP is a lasting enhancement of synaptic potentials elicited by repetitive stimulation at high frequencies observed in several pathways in the hippocampus and many other brain areas (for review, see Malenka and Nicoll, 1999). LTP is a potential model for the type of rapid synaptic modification thought to be necessary to form the associations between neuronal populations that underlie learning (Brown et al., 1988). There have been many reports of learning-induced plasticity, and the blockade of LTP, via infusion of NMDA receptor antagonists, has been shown to impair learning in spatial and nonspatial memory tasks (for review, see Martin and Morris, 2002). The large-amplitude, low-frequency (3-12 $\mathrm{Hz}$ ) theta rhythm is present only during certain learning-related behaviors (sniffing, exploring, rearing, and attending to behaviorally relevant stimuli) (Green and Arduini, 1954; Vanderwolf, 1969; Macrides et al., 1982), and learning is enhanced during these theta states (Winson, 1978; Berry and Seager, 2001; Hasselmo et al., 2002). The observation of theta rhythm in the local

Received June 2, 2003; revised 0ct. 18, 2003; accepted 0ct. 18, 2003.

This work was supported by National Institute of Mental Health Grants R01 MH60013 and R01 MH61492.

Correspondence should be addressed to James M. Hyman, Center for Memory and Brain, Department of Psychology, Boston University, 64 Cummington Street, Boston, MA 02215. E-mail: hyman@bu.edu.

Copyright $\odot 2003$ Society for Neuroscience $\quad$ 0270-6474/03/2311725-07\$15.00/0 field potential is reflective of the functional interactions of neurons throughout the hippocampus. Research into the interaction of theta and LTP has begun to elucidate a complex interplay between these two phenomena that spans the gap between the function of individual cells and the full hippocampal circuit.

An early link between theta activity and LTP was the observation that trains of stimuli delivered at intervals equal to theta frequencies more readily induced LTP than similar stimulation at other frequencies (Larson and Lynch, 1986; Greenstein et al., 1988).

Pavlides et al. (1988) demonstrated in urethane anesthetized rats that LTP was more effectively induced in the dentate gyrus when a tetanus was delivered on positive phases of theta. Similar results have been found in freely moving animals with stimulation of the perforant path (Orr et al., 2001). Holscher et al. (1997) elicited LTP by delivering tetanic bursts to the peak of the theta wave recorded locally in stratum radiatum in region CA1 during spontaneous theta in anesthetized animals. When similar tetanic bursts were delivered to the negative or zero phase of theta, there was no change in unpotentiated evoked potential slope.

A recent neural network model has been used to understand the relationship between function at the cellular and regional level in the hippocampus, by looking at the relationship between LTP and theta (Hasselmo et al., 2002). This model postulates that theta is associated with changes in circuit dynamics of hippocampal populations alternating between periods of encoding and retrieval. The difference between encoding and retrieval dynamics 
in the model includes changes in synaptic plasticity to provide a period of more LTP (on the peak of the wave in area CA1) and a period of less LTP or long-term depression (LTD) (on the trough of the wave in area CA1). The model requires precise timing of hippocampal LTP induction relative to the magnitude of synaptic transmission at the perforant path input and Schaffer collaterals, to keep memory traces from being corrupted. For instance, if the inputs from the perforant path are active at the same time that recurrent CA3 and Shaffer collateral inputs are active and LTP is maximal, then postsynaptic activity would combine a new input pattern from entorhinal cortex with the old pattern retrieval in CA3. In this case, LTP would result in spurious associations between new inputs and retrieved memories, which did not occur in conjunction with the new input. Our study set out to determine the role that phases of theta play in synaptic plasticity, by examining the effects of tetanic stimulation in region CA1 during the peak or trough of local theta activity in a behaving rat.

\section{Materials and Methods}

Subjects. Nineteen adult male Long-Evans rats, obtained from Charles River (Wilmington, MA), were used. The animals were individually housed and kept in a $12 \mathrm{hr}$ light/dark cycle with water available ad libitum. During training and after recovery from surgery, animals were maintained at $90 \%$ free-feeding weight.

Surgery. Animals were deeply anesthetized under halothane gas and pretreated with atropine $(0.1 \mathrm{mg} / \mathrm{kg})$ to prevent excessive salivation. Rats were placed in a David Kopf Instruments (Tujunga, CA) stereotaxic frame, and holes were drilled into the skull using a David Kopf Instruments stereotaxic drill. Bipolar stimulating electrodes mounted in a moveable drive were implanted ( $-3.5 \mathrm{~mm}$ posterior to bregma; \pm 2.5 $\mathrm{mm}$ from the midline). The stimulating bundle consisted of two $40-\mu \mathrm{m}-$ thick tungsten wires cut to equal length. The two wires extended $2.0 \mathrm{~mm}$ from the bottom of a 27 gauge thin-walled cannula that extended $1.0 \mathrm{~mm}$ from the bottom of the driver. A three-wire recording electrode $(40 \mu \mathrm{m})$ bundle (with wires separated in depth by a total of $0.5 \mathrm{~mm}$; extending a total of $2.0 \mathrm{~mm}$ from the 27 gauge thin-walled cannula) mounted in a moveable drive was implanted contralaterally to the stimulating electrode in area CA1 $(-3.5 \mathrm{~mm}$ posterior to bregma; $\pm 2.5 \mathrm{~mm}$ from the midline). Two of the five stainless steel bone screws used for mounting the head stage to the skull were used as indifferent grounds. The two drivers and EEG electrodes were mounted to the skull using dental acrylic. The electrodes were connected to a 10 pin Microtech (Belmont, $\mathrm{CA})$ plug that was also mounted above the skull. The entire apparatus was then shielded in plastic sheeting to prevent damage to the implant. After surgery, rats were orally treated with nonsteroidal antiinflammatories and antibiotics as needed. Animals were allowed 2 weeks of postoperative recovery.

Electrophysiology. The stainless steel recording wires passed the signal through preamplifier field effector transistors (NB Labs, Denison, TX) and an amplifier (Neuralynx, Tucson, AZ) before being passed to a Data Translation (Marlboro, MA) analog-to-digital board for storage by Experimenter's Workbench software (from DataWave Technologies, Broomfield, CO) running on a personal computer workstation. Evoked response signals were filtered between $1 \mathrm{~Hz}$ and $9 \mathrm{kHz}$ and were digitally sampled at $20 \mathrm{kHz}$. Stimulation was generated by a Grass Instruments (Quincy, MA) PSIU6 stimulus isolation unit driven by a Grass Instruments S88 stimulator. A dual-window discriminator (Bak Electronics, Germantown, MD) was used to tune tetanus bursts to particular phases of theta. The discriminator created two windows with variable widths (time span) and independently adjustable heights (amplitude) on a standalone oscilloscope (Fig. 1). The time offset between the two windows was also adjustable. By adjusting the windows to fit each individual animal's typical theta frequency and amplitude, we were able to tetanize on the peak or trough of theta accurately. If the input waveform successfully passed through both windows, a trigger was immediately sent to the stimulator. The delivery of the tetanus could then be manipulated, via digital delay on the stimulator, to fall on the next peak or trough. The

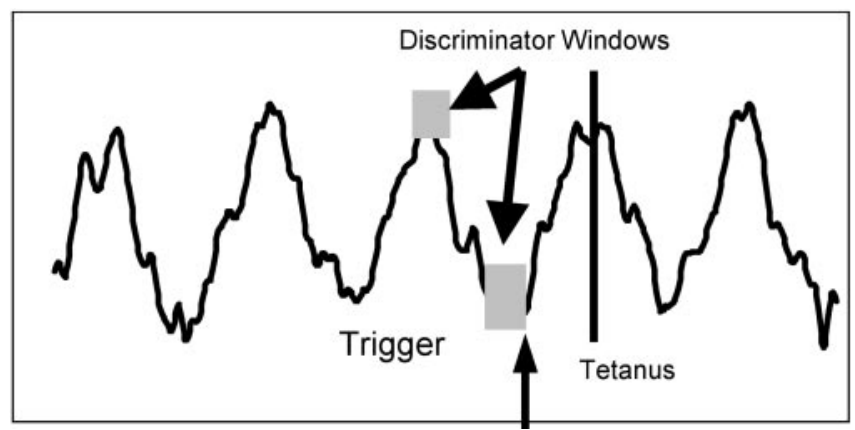

Figure 1. Dual-window discrimination. Dual-window discrimination used for detecting theta and delivering the tetanus to the appropriate phase. The discriminator created two windows with variable widths (time span) and independently adjustable heights (amplitude) on a standalone oscilloscope. The time offset between the two windows was also adjustable. By adjusting the windows to fit each individual animal's typical theta frequency and amplitude, we were able to tetanize on the peak or trough of theta accurately. If the input waveform successfully passed through both windows, a trigger was immediately sent to the stimulator. The delivery of the tetanus could then be manipulated, via digital delay on the stimulator, to fall on the next peak or trough. The appearance of the tetanic stimulation on the EEG provided accurate phase information for later analysis.

appearance of the tetanic stimulation on the EEG provided accurate phase information for later analysis.

Behavior and experimental protocol. Animals underwent 1-2 weeks of handling and running wheel training before surgery. Animals were reinforced (Froot Loops; Kelloggs, Battle Creek, MI) after 10-20 sec bouts of steady running on the wheel. One week after surgery, animals were anesthetized under halothane gas as the moveable electrodes were lowered into the hippocampus. Vertical placement of both recording and stimulating bundles was at first confirmed to be in stratum radiatum by the appearance of spontaneous theta activity (some animals required two sessions of lowering). After theta was observed, smaller adjustments were made in vertical placement of the stimulating and recording electrode bundles by examining potentials evoked by stimulation. In most cases, it was necessary to lower the stimulating bundle more than the recording electrodes, and, in some animals, stimulation electrode tracks were found to enter CA3. The electrode depths were adjusted to elicit the maximal, negative potential with no population spike contamination in the peak. The initial slope of the evoked potential was presumed to be the result of either antidromically activated CA3 neurons exciting CA1 through commissural connections or monosynaptic stimulation of contralateral CA3 neurons sending commissural connections to the recording site. Previous reports have shown little difference between contralateral and ipsilateral CA1 stimulation (Leung and Shen, 1995), and a recent report combines ipsilateral and contralateral potentials for analysis (Leung et al., 2003). Wyble et al. (2000) explicitly tested the difference between contralateral stratum radiatum and contralateral CA3 stimulation in evoked potentials recorded in stratum radiatum and found no significant differences between stimulation sites in slope or amplitude. Similar findings by Kaibara and Leung (1992) showed little difference in LTP induction. Other reports have used peak amplitude as a measure of contralateral stimulation in the dentate gyrus (Levy and Steward, 1979). The rather fast time course of the potentials recorded $(<8 \mathrm{msec}$ from stimulation) presumably rules out polysynaptic activation via the entorhinal cortex. Previous studies have shown that ipsilateral entorhinal cortical effects on the recorded potential caused by contralateral CA3 stimulation take between 18 and $22 \mathrm{msec}$ to appear (Deadwyler et al., 1975).

Two weeks after surgery, animals were given another wheel running training session. After rats showed consistent wheel running, they were anesthetized under halothane gas, and the moveable drives were manipulated to find a substantial and stable evoked potential (as described previously). After at least $10 \mathrm{~min}$ of normal awake behavior and normal EEG, an input-output curve was recorded to determine the amplitude of maximum current stimulation (defined as the smallest current stimula- 
tion required to evoke a field potential of asymptotic maximum amplitude).

A baseline field potential was recorded once every $20 \mathrm{sec}$ for a total of $30 \mathrm{~min}$. Test pulses consisted of stimulation $(250 \mu \mathrm{sec})$ at $70 \%$ of the amplitude necessary to induce maximum evoked field potential amplitude. During baseline recordings, rats were placed in a large recording chamber ( $62 \mathrm{~cm}$ long, $38 \mathrm{~cm}$ high, $36 \mathrm{~cm}$ deep). During the periods of test potential collection, animals were in the same high-walled chamber both before and after tetanization, to help ensure that the animals behavior was similar during both periods. Within the high-walled chamber, animals received no external sensory stimuli, and, for the most part, they sat quietly. Test pulses fell during nontheta activity or on random phases of theta. Previous work from this laboratory (Wyble et al., 2000) has shown random stimulation to fall evenly on different periods of theta, and no net difference was apparent in the numbers of stimuli falling on a particular phase. After establishment of a steady baseline, rats were moved to the wheel chamber for tetanization. During extended bouts $(10-15 \mathrm{sec})$ of running, a tetanic burst was delivered to either the peak or trough of the local theta wave (depending on experimental condition; see below). The tetanus consisted of three bursts of current stimulation at $90 \%$ of maximum evoked field potential amplitude. Each burst was made up of five $150 \mu \mathrm{sec}$ pulses delivered at $400 \mathrm{~Hz}$ (burst width was $12.5 \mathrm{msec}, \sim 36^{\circ}$ of a cycle of theta) to allow the delivery of five pulses in a time as short as possible to ensure that the bursts only fell on one unambiguous phase of theta. We chose to use five bursts of tetanic stimulation to keep the methodology similar to previous studies by Orr et al. (2001), who used two to four pulses at $400 \mathrm{~Hz}$, and Holscher et al. (1997), who used five pulses at $200 \mathrm{~Hz}$. Bursts were separated by at least $2-3$ sec to reduce the likelihood of seizure activity. After successful delivery of the tetanic bursts, animals were placed back into the large recording chamber, and field potentials were elicited every $20 \mathrm{sec}$ for $30-60 \mathrm{~min}$ using the baseline test pulse current.

Experimental conditions. One-half of the animals were initially tetanized on the positive peak of theta, and the other one-half were initially tetanized on the negative trough of the wave. After at least $5 \mathrm{~d}$ had passed and it had been measured that evoked potentials had returned to baseline levels, the animals were run under the other condition. Because our stimulation protocol used only a small number of bursts delivered in a short time period, we did not expect titanic-induced changes to persist as long has been documented previously by Racine et al. (1983). After each session, the unfiltered EEG was visually analyzed to determine how accurately each burst was delivered to the proper phase of theta. Some animals $(n=6)$ were run two to three times on one condition. This was attributable to different reasons, such as the animal becoming unplugged, but in most cases, it was because of inaccurate tetanic stimulation delivery (mostly on trough stimulation sessions). Before being run again, all animals were rested for at least $5 \mathrm{~d}$, and evoked potentials were recorded to ensure that a steady baseline was present. The trough of theta tended to be more difficult to accurately stimulate with three tetanic bursts than the peak. Because of this, some animals were only used for one of the conditions (only trough, $n=3$; only peak, $n=3$; both conditions, $n=7$ ). We believe the trough was harder to tetanize because of the scalloped shape of the unfiltered theta wave found in behaving animals. We were strict to only use the trough (true negative point of each cycle) rather than just any point at which the waveform was negative. Using this definition, the trough was a considerably smaller amount of time than the peak (the trough was approximately $\approx 75^{\circ}$ of a theta cycle or $\sim 25$ msec, whereas the peak was approximately $\approx 150^{\circ}$ or $\sim 50 \mathrm{msec}$ ), thus making three accurate trough stimulations ( $12.5 \mathrm{msec}$ each) in a single session much more difficult.

Histology. After each animal had completed the experiment, they were deeply anesthetized under halothane gas and were electrolytically lesioned at each electrode location. They were then perfused with a solution containing 25 parts 10\% buffered Formalin, 1 part glacial acetic acid, and $10 \mathrm{gm}$ of potassium ferrocyanide. This solution causes a Prussian blue reaction, which marks with blue the location of the iron particles deposited by the electrode lesion. The brains were then removed and stored in a $10 \%$ buffered Formalin solution for $\sim 1-2$ weeks. After this time, the brains were then sliced, mounted, and stained with neutral red to determine precise electrode locations.

\section{Results}

In most animals, delivery of tetanic burst stimulations was confirmed to be close to either the peak $(n=8)$ or trough $(n=7)$ of the local theta wave. Post hoc analyses revealed which experiments did not receive the correct stimulation phase. In these experiments, at least one of the three tetanic bursts fell on an ambiguous phase of theta. These data were excluded from the experiment, animals were rested for at least $5 \mathrm{~d}$ before evoked potentials were again measured to ensure a steady baseline, and the animals were run again on the experiment. Post-run analyses of the EEG record confirmed that all animals included in the analysis had received precisely three tetanic bursts on the peak or trough, respectively. Examples of stimulation at three peaks are shown in Figure $2 \mathrm{~A}$, and examples of stimulation at three troughs are shown in Figure $2 B$. Because of the relative difficulty of delivering three bursts to the trough of theta accurately in one session, trough deliveries were more closely analyzed to determine their true phase relationships. Figure $2 C$ shows an average of all trough stimulations centered around the first pulse of each high-frequency burst. The averaged waveforms show two to three cycles at theta frequency (7-9 Hz) preceding the first pulse, indicating that all stimulations of the trough used in the analysis were indeed delivered during the most negative point of the wave (trough).

Tetanic bursts delivered to the peak of theta activity elicited a mean increase of $14.6 \pm 0.85 \%$ in amplitude of potentials and a $17.9 \pm 0.94 \%$ increase in slope of potentials for $30 \mathrm{~min}$ after stimulation (Fig. 3). $t$ tests revealed significant differences between evoked potentials after tetanus and baseline evoked potentials in amplitude $(t=12.25 ; p<0.0001 ; \mathrm{df}=2)$ and slope $(t=$ 17.05; $p<0.0001 ; \mathrm{df}=2)$.

Tetanic bursts delivered to the trough of theta activity resulted in a mean decrease of $10.6 \pm 1.7 \%$ in potential amplitude and a decrease of $12.9 \pm 1.03 \%$ in potential slope averaged over the 30 min after stimulation (Fig. 3). $t$ tests revealed significant differences between evoked potentials after tetanus and baseline evoked potentials in amplitude $(t=6.53 ; p<0.0001 ; \mathrm{df}=2)$ and slope $(t=10.5 ; p<0.0001$; df $=2)$.

Figure $3 A$ shows the average change in evoked potential slope and amplitude from baseline for all animals for $30 \mathrm{~min}$ of test pulses after tetanic stimulation. Figure $3 B$ shows the change in evoked potential amplitude for all peak and trough sessions for the entire course of the sessions. The data points represent averages for all animals for 4 min time periods shown in percentage change from baseline. The time course data show the robust effects the tetanic stimulation had on evoked potential amplitude in the first test pulse after stimulation and for a $30 \mathrm{~min}$ time period afterward. The triangles show the trough stimulation sessions, and the squares show the peak stimulation sessions. Both session types received the same tetanic burst stimulation, with the only difference being the phase of the theta wave on which the bursts were delivered.

Figure $4 A$ shows a representative example of tetanic stimulation during the peak of theta rhythm. The data points at the bottom are the slope of potentials generated by test pulses every $20 \mathrm{sec}$. The arrow shows when tetanic stimulation was delivered, and data points after that stimulation show a significant increase in slope. Averaged evoked potential waveforms are shown above for both the baseline period (left) and the post-tetanus period (right). Figure $4 B$ shows a representative example of tetanic stimulation during the trough of theta rhythm. As in the other exam- 


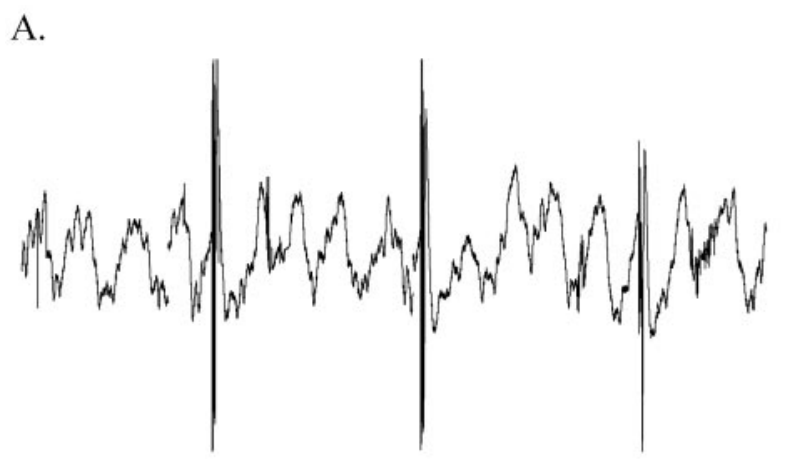

B.

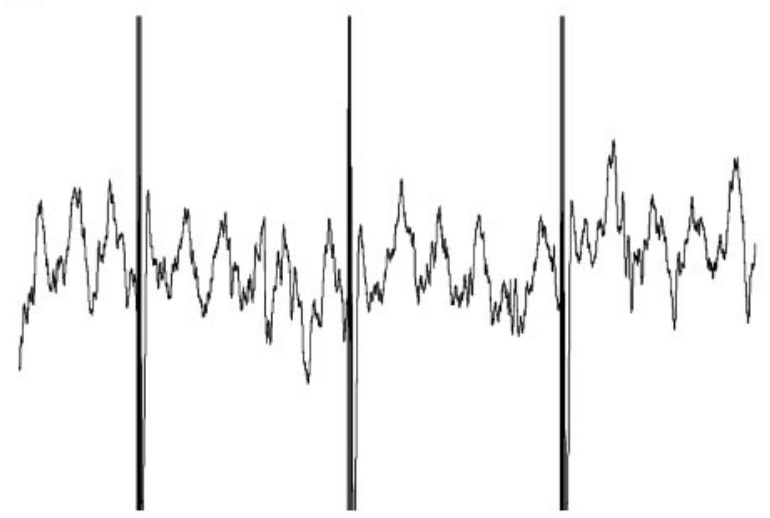

C.

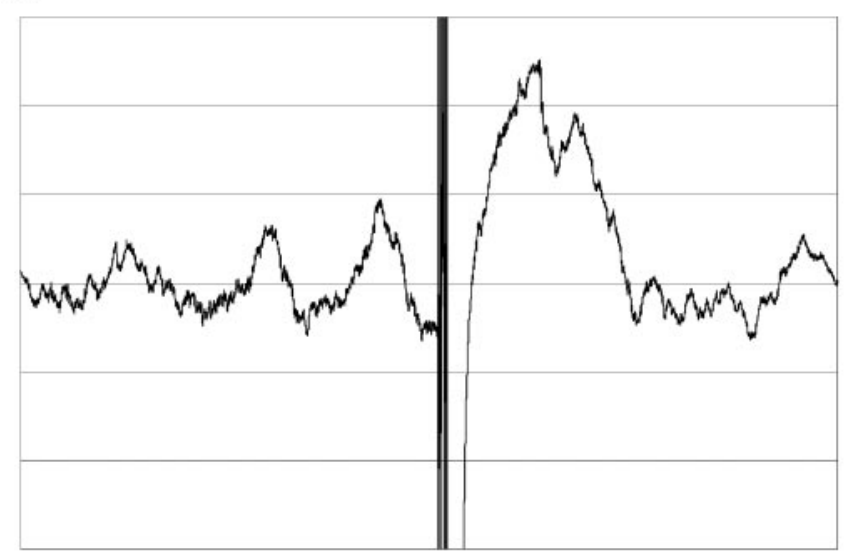

Figure 2. Tetanus delivery. $A, B, A$ representative example of three high-frequency bursts delivered to the peak of the theta wave $(A)$ and to the trough of the theta wave $(B)$. C, The graph shows an average of the EEG ( $n=21 ; 3$ bursts per 7 animals) for 500 msec, centered on the first pulse of the tetanic burst. The average reveals two to three cycles of a theta rhythm before the tetanic burst, indicating a consistent phase relationship for each tetanic burst.

ple, data points at the bottom are evoked potential slope for test pulses during the baseline and post-tetanus periods. Both the slopes and the averaged waveforms above the data points show a clear and significant decrease after trough tetanic stimulation.

A small group of animals had test potentials collected for 60 min after tetanic stimulation to explore the duration of the evoked potential changes. Single-factor $t$ tests were performed for $10 \mathrm{~min}$ bins and demonstrated that the change in evoked potentials remained significant for every bin over the full $60 \mathrm{~min}$. Even the last bin from 50-60 min showed significant increases for peak stimulation sessions $(n=4)$ for both amplitude of evoked potentials (18.4\% increase from baseline; $t=5.35 ; p<0.0001 ; \mathrm{df}=2$ )
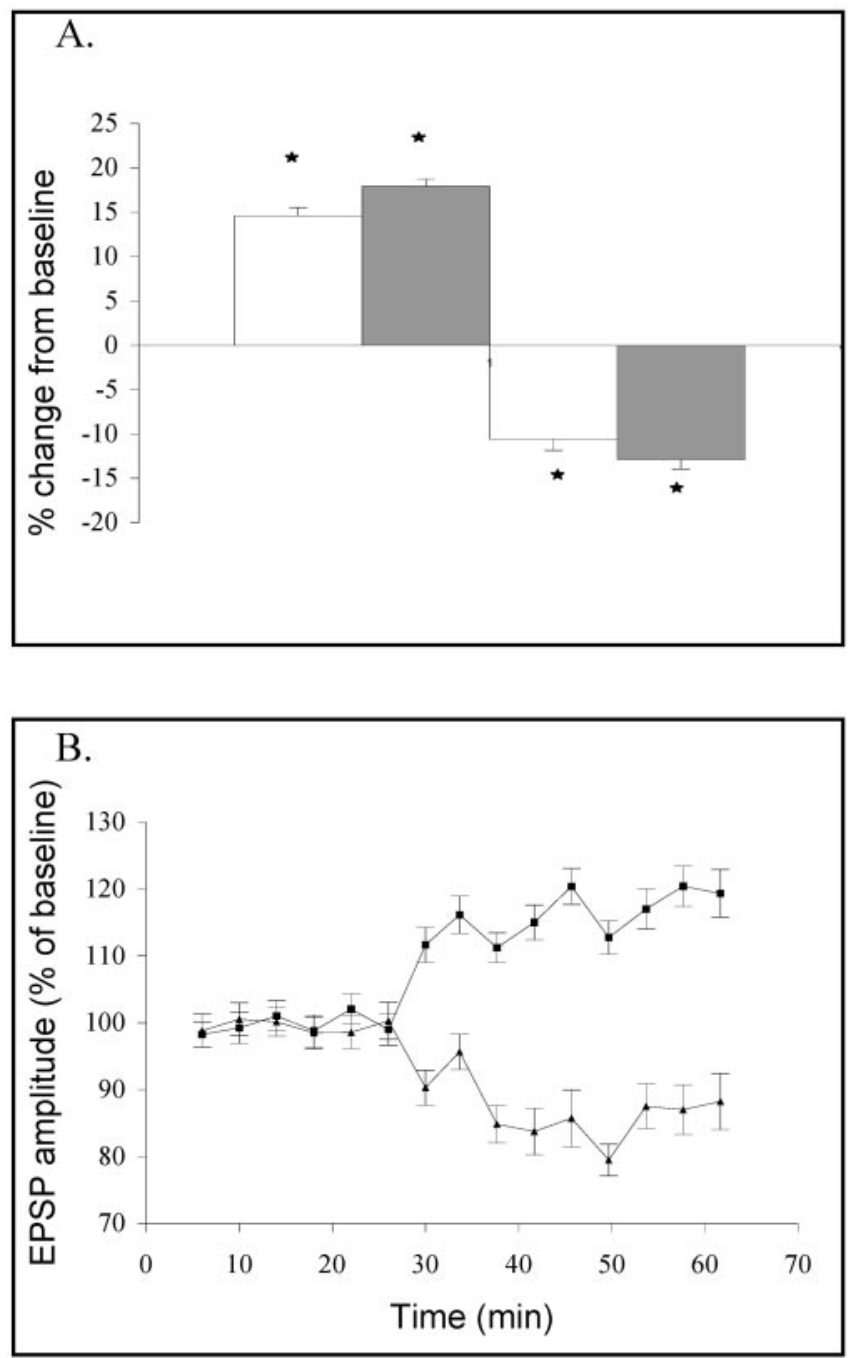

Figure 3. A, Effects of peak or trough tetanic stimulation on group means for slope (shaded) and amplitude (white). Series 1 and 2 are for peak stimulation sessions $(n=8)$, and series 3 and 4 are for trough stimulations $(n=7)$. Error bars are SE $\left({ }^{*} p<0.001\right) . B$, The effects of peak or trough tetanic stimulation on amplitude group means for a $30 \mathrm{~min}$ baseline period and $30 \mathrm{~min}$ after delivery of three tetanic bursts. Peak stimulation sessions are shown with squares, and trough stimulation sessions are shown with triangles. Peak stimulation sessions consistently revealed a consistent increase in evoked potential amplitude for $30 \mathrm{~min}$ after the tetanic bursts, whereas trough stimulation consistently revealed a lasting depression of the evoked potential amplitude for $30 \mathrm{~min}$ after the tetanic bursts. Each data point represents the average across animals for 4 min periods, normalized to percentage change from baseline.

and for slope of evoked potentials (19.9\% increase from baseline; $t$ test results for evoked potentials taken between minutes 50 and $60, t=5.16 ; p<0.0001$; df $=2$ ). Similarly, $t$ tests showed that significant decreases for trough stimulation sessions $(n=2)$ were maintained over the course of $60 \mathrm{~min}$ in both amplitude of evoked potentials ( $14.9 \%$ decrease from baseline; $t$ test results for evoked potentials taken between minutes 50 and $60, t=10.91$; $p<0.0001 ; \mathrm{df}=2)$ and slope of evoked potentials $(17.2 \%$ decrease from baseline; $t$ test results for evoked potentials taken between minutes 50 and $60, t=5.84 ; p<0.0001$; $\mathrm{df}=2$ ).

\section{Discussion}

These results show that induction of LTP and LTD at the Schaffer collaterals in region CA1 of the hippocampus is sensitive to the phase of the naturally occurring locomotion-induced theta rhythm. Identical stimulation (three bursts of five pulses at 400 
A.

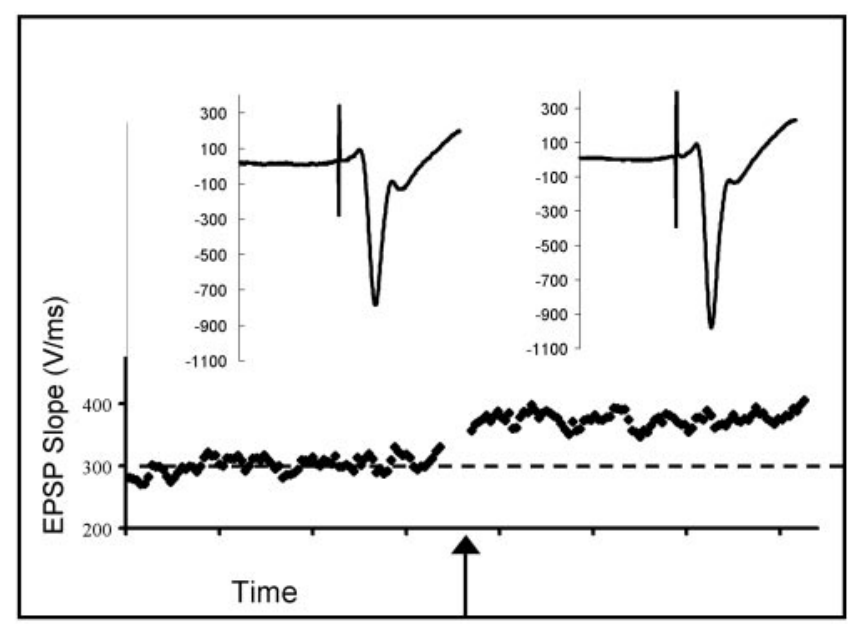

B.

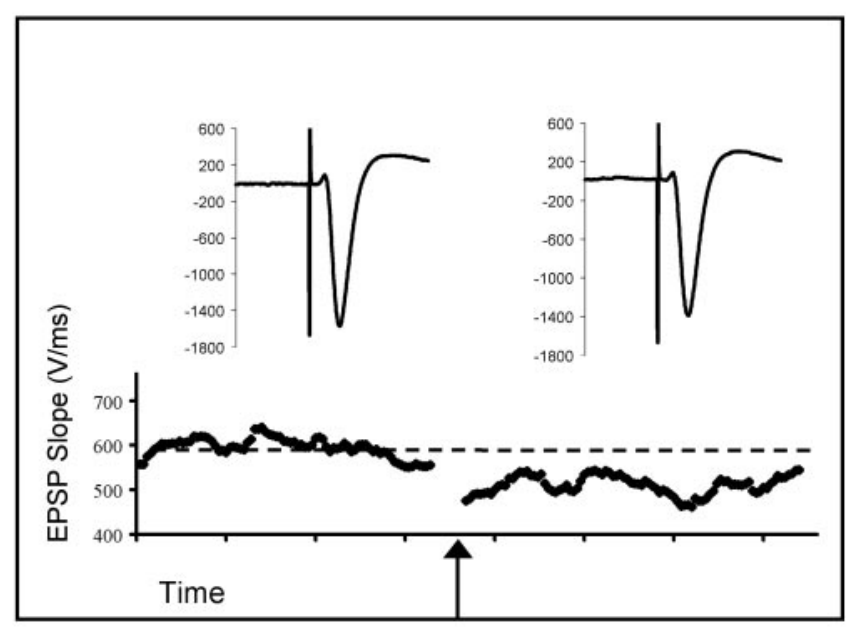

Figure 4. Representative examples of the effect of peak and trough tetanic stimulation. $A$, The graph shows the change in synaptic potential caused by three bursts of high-frequency stimulation to the peak of theta. Points are evoked potential slope (volts per millisecond) taken once every $20 \mathrm{sec}$ for $30 \mathrm{~min}$ before and after tetanic stimulation (total time plotted, $60 \mathrm{~min}$ ). The dotted line is the average evoked potential slope for the baseline-pretetanus period. In the inset are averaged waveforms for the baseline period (left) and the post-tetanus period (right). In this representative session of peak tetanic stimulation, the averaged waveforms show a clear difference in evoked potential slope and peak amplitude, with the post-tetanic potentials having a steeper slope and larger peak. $B$, The graph shows the results of three bursts of highfrequency stimulation to the trough of theta. Again, points are evoked potential slope (volts per millisecond) taken at the same time intervals. The dotted line is the average evoked potential slope for the baseline-pretetanus period. The inset shows average waveforms for baseline (left) and post-tetanus (right) periods. For this representative session of trough tetanic stimulation, the post-tetanic potentials were consistently lower in peak amplitude and slope, and those differences can be seen in the averaged waveforms. The arrows show the point in time the tetanic bursts were delivered.

$\mathrm{Hz}$ ) delivered to either the peak or trough of the local theta wave resulted in opposing changes in evoked potentials. Stimulation to the peak produced LTP, resulting in increases in the slope and amplitude of the evoked potential that persisted for at least 60 min. The delivery of three tetanic bursts to the trough of theta reliably yielded significant decreases in both amplitude and slope of evoked potentials (LTD), which remained steady for at least 30 min.

These results are consistent with studies examining the effects of perforant path tetanic stimulation on specific phases of local theta (Pavlides et al., 1988; Orr et al., 2001). The current results were recorded in stratum radiatum of region CA1, but the exact depth of those recordings cannot be determined with great accuracy. Depth profiles reveal an incomplete reversal between region CA1 and the dentate gyrus (Buzsaki et al., 1983), showing a gradual shift in phase occurring between CA1 pyramidal layer through stratum radiatum until the fissure. The dentate gyrus is in-phase with fissure theta, and current source density studies show that the current sink in stratum radiatum is $\sim 180^{\circ}$ out-ofphase with the sink in stratum lacunosum-moleculare (Brankack et al., 1993), and the local EEG in radiatum is substantially outof-phase with the fissure theta (Buzsaki et al., 1983; Brankack et al., 1993). Therefore, the results presented here suggest that greater LTP induction at the peak of local theta in stratum radiatum EEG corresponds to LTP induction closer to the trough of theta at the fissure.

Previous reports on the best phase of LTP induction examined potentiation at the perforant path of the hippocampus, which was seen in evoked potentials recorded from the hilus (Orr et al., 2001) and the granule cell layer (Pavlides et al., 1988). Thus, those previous studies analyzed the induction of LTP at hippocampal afferents rather than the Schaffer collaterals. These afferents also appear to have best LTP induction at the peak of local theta rhythm, corresponding to when local transmission is the weakest. This implies that the perforant pathway inputs have a similar associative function as that proposed for the Schaffer collaterals. It is possible that the LTP induction in those previous studies was predominantly at synapses of the middle molecular layer, which receive input from the medial entorhinal cortex. There is evidence that units in the medial and lateral entorhinal cortex fire out-of-phase with one another (Stewart et al., 1992) (for discussion, see Brankack et al., 1993). It should also be noted that the studies using recordings in the dentate gyrus contain a similar level of uncertainty about the phase relationship between the local theta recorded in the study and the phase of fissure theta (which is a standard reference in theta studies).

Our results support the previous results from anesthetized preparations (Holscher et al., 1997), which found LTP to be preferentially induced in area CA1, after tetanic bursts on the peak of sensory-induced (tail pinch) theta rhythm. These results are similar to the present findings in that both experiments used a relatively small tetanic stimulus and found similar changes in evoked potentials. However, in that previous study, when bursts were delivered during negative phase of theta, it did not produce any significant change from baseline, unpotentiated levels in potential amplitude, or slope (Holscher et al., 1997). However, they did not focus on delivering stimulation strictly to the trough, allowing the stimulation to fall anywhere in the negative phase. The present experiment only includes sessions in which stimulation was delivered successfully three times to the trough of the theta rhythm (we defined trough as the lowest point on the theta wave before the waveform starts to rise again). We were very strict in our post-session analysis of the EEG to ensure that each tetanic burst arrived at the point at which the wave stopped falling (Fig. 2C).

This is the first time LTD (depression, not depotentiation) of evoked potentials has been reported in the hippocampus without using low-frequency stimulation. The bursts of high-frequency stimulation used in the current experiment more closely resemble the natural bursting activity of neurons found in the hippocampus. A problem with long-term depression research has been that only prolonged periods (5-10 min) of low-frequency 
stimulations (Dudek and Bear, 1992) have produced the depression, and stimulation of this sort has not been found to exist endogenously in the hippocampus (Huerta and Lisman, 1995).

Work from slice physiology has revealed that tetanic stimulation applied during higher intracellular membrane potentials and dendritic $\mathrm{Ca}^{2+}$ levels leads to LTP and that stimulation results in LTD when these levels are lower yet still elevated (Cummings et al., 1996). Kamondi et al. (1998) showed putative $\mathrm{Ca}^{2+}$ spikes phase locked to the positive phase of local field theta in CA1 pyramidal layer and that these spikes only occurred after a sufficient amount of membrane depolarization. Other research has shown that the effect of local dendritic spikes on LTP was great enough to overcome somatic hyperpolarization (Golding et al., 2002), further underscoring the importance that voltagegated dendritic $\mathrm{Ca}^{2+}$ channels possess in LTP induction dynamics phenomena and those described by Cummings et al. (1996) could account for the present results. Within such a model, during the peak of the local field potential, $\mathrm{Ca}^{2+}$ levels are large enough at the dendrites of CA1 pyramidal to elicit high-threshold spikes and are therefore likely to be high enough to create the appropriate dynamics for LTP (as described by Cummings et al., 1996). As the theta wave reaches the trough in CA1, the intracellular membrane potential of dendrites also falls (Kamondi et al., 1998), presumably associated with lower (yet still elevated) dendritic $\mathrm{Ca}^{2+}$ levels, creating the appropriate dynamics for depression. It is possible that the theta rhythm is associated with these alternating states of dendritic $\mathrm{Ca}^{2+}$ dynamics on different phases, providing the mechanism necessary in Lisman's (1989) model of synaptic plasticity. It is possible that the relationship between dendritic $\mathrm{Ca}^{2+}$ levels, fluctuating intracellular membrane potentials, and theta rhythm field oscillations will explain the results presented in the current study.

The present results may support the model of hippocampal functioning put forward by Hasselmo et al. (2002), which emphasizes how the oscillating change in extracellular field potential of the theta rhythm could indicate changing dynamics of circuit function. The rhythmic EEG is accompanied by oscillatory changes in synaptic transmission at different synaptic inputs. In area CA1 when the pyramidal layer theta rhythm is at its peak, synaptic input from the entorhinal cortex is at its greatest (Brankack et al., 1993). At this time, pyramidal cells in CA3, which give rise to recurrent synapses in CA3, and CA3-to-CA1 connections fire at their lowest rates (Fox et al., 1986). These factors, along with the present results (showing strong LTP at the peak of the local theta wave in stratum radiatum), create the appropriate dynamics for encoding of new memory traces in areas CA3 and CA1. In this model, modification of CA3 synaptic input to the dendrites will depend on dendritic depolarization caused by entorhinal input but not CA3 input. When the theta wave in area CA1 is at its trough, synaptic input from the entorhinal cortex is weakest, whereas recurrent synapses in CA3 and connections from CA3-to-CA1 are most active. If LTP was possible during the trough of CA1 theta when input from CA3 and output from CA1 to the subiculum are the strongest, then the new encoded memory trace could suffer from interference from previously stored associations (as seen in the computational model by Hasselmo et al., 2002).

The computational model by Hasselmo et al. (2002) incorporates a $180^{\circ}$ phase reversal between area CA1 pyramidal cell layer theta rhythm and fissure theta. As discussed previously, all data used in the current experiment was confirmed to come from stratum radiatum. It is possible that the EEG recorded was somewhat out-of-phase with CA1 pyramidal layer theta so that the peak of recorded EEG might not have been the true peak of the wave. It may be that the shift may be only $100^{\circ}$ from fissure theta instead of $180^{\circ}$. In that case, the induction of LTD (or the least LTP) might be $100^{\circ}$ after strongest entorhinal cortex input, and the peak induction of LTP might be $80^{\circ}$ before the weakest entorhinal cortex input. This time phase of LTP might fit with the offset of inhibitory interneurons relative to the peak of fissure theta (Stewart et al., 1992). It is also possible that the peak and trough measured by the recording electrodes do not represent the best and worst phase for LTP induction but rather the recordings tapped into high and low points of LTP induction slightly offset from the maximum and minimum points.

The present results reveal a clear relationship between the timing of tetanic stimulation relative to the phase of theta rhythm oscillations and synaptic plasticity. Identical burst stimulation elicited either a marked increase or decrease in evoked potentials in stratum radiatum, depending solely on the phase of local theta activity. Future studies in hippocampal slices could help determine the extent of the relationship between dendritic $\mathrm{Ca}^{2+}$ levels, phase of the theta rhythm, and the outcome of tetanic stimulation. Also, future experimentation in which fissure theta is recorded during theta phase-dependent tetanic burst delivery could help determine the specific phase relationships present in both stratum radiatum and the dentate gyrus. These findings provide support for the theta theory by Hasselmo et al. (2002), suggesting the importance of maintaining strict phase relationships between synaptic inputs and the induction of LTP and LTD.

\section{References}

Berry SD, Seager MA (2001) Hippocampal theta oscillations and classical conditioning. Neurobiol Learn Mem 76:298-313.

Brankack J, Stewart M, Fox SE (1993) Current source density analysis of the hippocampal theta rhythm: associated sustained potentials and candidate synaptic generators. Brain Res 615:310-327.

Brown TH, Chapman PF, Kairiss EW, Keenan CL (1988) Long-term synaptic potentiation. Science 242:724-728.

Buzsaki G, Leung LW, Vanderwolf CH (1983) Cellular bases of hippocampal EEG in the behaving rat. Brain Res 287:139-171.

Cummings JA, Mulkey RM, Nicoll RA, Malenka RC (1996) $\mathrm{Ca}^{2+}$ signaling requirements for long-term depression in the hippocampus. Neuron 16:825-833.

Deadwyler SA, West JR, Cotman CW, Lynch GS (1975) A neurophysiological analysis of commisural projections to the dentate gyrus of the rat. J Neurophysiol 38:167-184.

Dudek SM, Bear MF (1992) Homosynaptic long-term depression in area CA1 of hippocampus and effects of $N$-methyl-D-aspartate receptor blockade. Proc Natl Acad Sci USA 89:4363-4367.

Fox SE, Wolfson S, Ranck Jr JB (1986) Hippocampal theta rhythm and the firing of neurons in walking and urethane anesthetized rats. Exp Brain Res 62:495-508.

Golding NL, Staff NP, Spruston N (2002) Dendritic spikes as a mechanism for cooperative long-term potentiation. Nature 418:326-331.

Green JD, Arduini AA (1954) Hippocampal electrical activity in arousal. J Neurophysiol 17:533-557.

Greenstein YJ, Pavlides C, Winson J (1988) Long-term potentiation in the dentate gyrus is preferentially induced at theta rhythm periodicity. Brain Res 438:331-334.

Hasselmo ME, Bodelon C, Wyble BP (2002) A proposed function for hippocampal theta rhythm: separate phases of encoding and retrieval enhance reversal of prior learning. Neural Comput 14:793-817.

Holscher C, McGlinchey L, Anwyl R, Rowan MJ (1997) HFS-induced longterm potentiation and LFS-induced depotentiation in area CA1 of the hippocampus are not good models for learning. Psychopharmacology (Berl) 130:174-182.

Huerta PT, Lisman JE (1995) Bidirectional synaptic plasticity induced by a single burst during cholinergic theta oscillation in CA1 in vitro. Neuron 15:1053-1063.

Kaibara T, Leung S (1992) Basal versus apical dendritic long-term potenti- 
ation of commissural afferents to hippocampal CA1: a current-source density study. J Neurosci 13:2391-2404.

Kamondi A, Acsady L, Wang XJ, Buzsaki G (1998) Theta oscillations in somata and dendrites of hippocampal pyramidal cells in vivo: activitydependent phase-precession of action potentials. Hippocampus 8:244-261.

Larson J, Lynch G (1986) Induction of synaptic potentiation in hippocampus by patterned stimulation involves two events. Science 232:985-988.

Leung LS, Shen B (1995) Long-term potentiation at the apical and basal dendritic synapses of CA1 after local stimulation in behaving rats. J Neurophysiol 73:1938-1946.

Leung LS, Shen B, Rajakumar N, Ma J (2003) Cholinergic activity enhances hippocampal long-term potentiation in CA1 during walking in rats. J Neurosci 23:9297-9304.

Levy WB, Steward O (1979) Synapses as associative memory elements in the hippocampal formation. Brain Res 175:233-245.

Lisman J (1989) A mechanism for the Hebb and the anti-Hebb processes underlying learning and memory. Proc Natl Acad Sci USA 86:9574-9578.

Macrides F, Eichenbaum HB, Forbes WB (1982) Temporal relationship between sniffing and the limbic theta rhythm during odor discrimination reversal learning. J Neurosci 2:1705-1717.
Malenka RC, Nicoll RA (1999) Long-term potentiation-a decade of progress? Science 285:1870-1874.

Martin SJ, Morris RG (2002) New life in an old idea: the synaptic plasticity and memory hypothesis revisited. Hippocampus 12:609-636.

Orr G, Rao G, Houston FP, McNaughton BL, Barnes CA (2001) Hippocampal synaptic plasticity is modulated by theta rhythm in the fascia dentata of adult and aged freely behaving rats. Hippocampus 11:647-654.

Pavlides C, Greenstein YJ, Grudman M, Winson J (1988) Long-term potentiation in the dentate gyrus is induced preferentially on the positive phase of theta-rhythm. Brain Res 439:383-387.

Racine RJ, Milgram NW, Hafner S (1983) Long-term potentiation phenomena in the rat limbic forebrain. Brain Res 260:217-231.

Stewart M, Quirk GJ, Barry M, Fox SE (1992) Firing relations of medial entorhinal neurons to the hippocampal theta rhythm in urethane anesthetized and walking rats. Exp Brain Res 90:21-28.

Vanderwolf CH (1969) Hippocampal electrical activity and voluntary movement in the rat. Electroencephalogr Clin Neurophysiol 26:407-418.

Winson J (1978) Loss of hippocampal theta rhythm results in spatial memory deficit in the rat. Science 201:160-163.

Wyble BP, Linster C, Hasselmo ME (2000) Size of CA1-evoked synaptic potentials is related to theta rhythm phase in rat hippocampus. J Neurophysiol 83:2138-2144. 